\title{
Impact of substrate on tip-enhanced Raman spectroscopy: A comparison between field-distribution simulations and graphene measurements
}

\author{
Hudson Miranda $\odot,{ }^{1}$ Cassiano Rabelo $\odot,{ }^{1}$ Luiz Gustavo Cançado, ${ }^{2}$ Thiago L. Vasconcelos, ${ }^{3}$ Bruno S. Oliveira, ${ }^{3}$ \\ Florian Schulz, ${ }^{4}$ Holger Lange $\odot,{ }^{4,5}$ Stephanie Reich, ${ }^{6}$ Patryk Kusch $\odot,{ }^{6}$ and Ado Jorio $\oplus^{1,2}$ \\ ${ }^{1}$ Graduate Program in Electrical Engineering - UFMG - CEP: 31270-901, Belo Horizonte, MG, Brazil \\ ${ }^{2}$ Departamento de Física - UFMG - CEP: 31270-901, Belo Horizonte, MG, Brazil \\ ${ }^{3}$ Divisão de Metrologia de Materiais - Inmetro - CEP: 25250-020, Duque de Caxias, RJ, Brazil \\ ${ }^{4}$ Institute for Physical Chemistry, University of Hamburg, Martin-Luther-King Platz 6, 20146 Hamburg, Germany \\ ${ }^{5}$ The Hamburg Centre for Ultrafast Imaging, 22761 Hamburg, Germany \\ ${ }^{6}$ Department of Physics, Freie Universität Berlin, Arnimallee 14, D-14195 Berlin, Germany
}

(Received 17 January 2020; revised manuscript received 23 May 2020; accepted 9 June 2020; published 29 June 2020)

\begin{abstract}
Tip-enhanced Raman spectroscopy (TERS) has reached nanometer spatial resolution for measurements performed at ambient conditions and subnanometer resolution at ultrahigh vacuum. Super-resolution (beyond the tip apex diameter) TERS has been obtained mostly in the gap mode configuration, where a conductive substrate localizes the electric fields. Here we present experimental and theoretical TERS to explore the field distribution responsible for spectral enhancement. We use gold tips of $40 \pm 10 \mathrm{~nm}$ apex diameter to measure TERS on graphene, a spatially delocalized two-dimensional sample, sitting on different substrates: (i) glass, (ii) a thin layer of gold and (iii) a surface covered with $12 \mathrm{~nm}$ diameter gold spheres, for which $6 \mathrm{~nm}$ resolution is achieved at ambient conditions. The super-resolution is due to the field configuration resulting from the coupled tip-sample-substrate system, exhibiting a nontrivial spatial surface distribution. The field distribution and the symmetry selection rules are different for nongap versus gap mode configurations. This influences the overall enhancement which depends on the Raman mode symmetry and substrate structure.
\end{abstract}

DOI: 10.1103/PhysRevResearch.2.023408

\section{INTRODUCTION}

Tip-enhanced Raman spectroscopy (TERS) is an optical imaging technique with a resolution far beyond the diffraction limit of light, which provides, simultaneously, scanning probe microscopy (SPM) and Raman spectroscopy information [1-15]. It is based on the illumination of a sharp metallic tip that, on one hand, concentrates the incoming exciting electromagnetic field to a nanoscale near-field at the tip apex and, on the other hand, collects the near-field Raman scattering from the sample, resulting in a localized and enhanced stimulation of the sample's scattering [16-19]. Therefore it is not uncommon to simplistically assume that the TERS characteristics, including imaging resolution, is defined solely by the tip apex structure. However, several TERS experiments have now shown resolutions far beyond the tip apex dimension, achieving the nanometer scale in air [20] and the angstrom scale in ultrahigh vacuum [21-23]. Such "super-resolution" has been obtained using special experimental procedures, like local tip-induced pressure [10], and in most cases utilizing

Published by the American Physical Society under the terms of the Creative Commons Attribution 4.0 International license. Further distribution of this work must maintain attribution to the author(s) and the published article's title, journal citation, and DOI. the so-called gap mode configuration, where the enhancement can be further increased by locating the sample between the tip and a flat metallic substrate [21,24,25]. Whereas in the conventional TERS configuration the field enhancement at the tip apex is conventionally due to the excitation of localized surface plasmon resonance on the tip shaft [26,27], the gap mode configuration makes use of the electric field enhancement by the gap-plasmon resonance that appears in the confined dielectric space between the tip end and the metallic substrate [28].

In this work, we study the spatial distribution of the field enhancement during TERS experiments in three different TERS configurations: regular (nongap mode), gap mode with a continuous metallic substrate, and a "structured" gap mode, utilizing regularly spaced metallic nanospheres as substrate. As a reference sample we utilize graphene, an efficient and two-dimensional Raman scatterer [29,30], which enables total surface sensing on top of the different substrates to show the significant influence of the substrate structure in the TERS results. We first introduce, in Sec. II, the technical aspects. In Sec. III, the experimental results are discussed, separated in three main findings: (A) TERS efficiency as a function of the tip-laser alignment within the focus; (B) TERS enhancement dependence on substrate structure and phonon symmetry; (C) the achievement of super-resolution for "structured" gap mode. We then focus, in Sec. IV, on how the "structured" gap mode is capable of generating an apparent super-resolution image. In Sec. V, we present the conclusions of this work. 

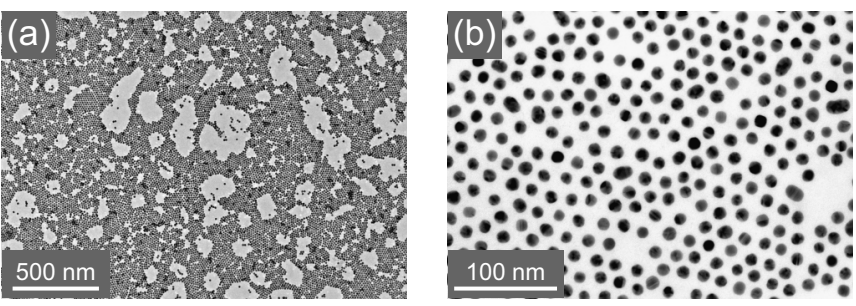

FIG. 1. Scanning electron microscopy (SEM) images of substrate (iii), i.e., oleylamine-stabilized gold nanoparticles, in two different magnification scales.

\section{TECHNICAL ASPECTS}

\section{A. Sample preparation}

Graphene, as a spatially delocalized two-dimensional TERS sample, was prepared by the mechanical exfoliation method and deposited on three different substrates: (i) glass; (ii) a $12 \mathrm{~nm}$ thick layer of gold evaporated on glass (Au film); and (iii) a surface of $12 \mathrm{~nm}$ diameter gold nanoparticles (AuNP) deposited on glass. The thickness of the gold film (substrate ii) was chosen to match the diameter of the AuNP.

The glass substrate (i) is a usual laboratory glass coverslip cleaned in a three step process involving acetone, isopropyl alcohol and deionized water. As for the gold film (ii), in order to get a homogeneous gold substrate with $12 \mathrm{~nm}$ thickness, gold is evaporated on a glass coverslip using the Nano 36 thermal evaporator from Kurt J-Lesker. It allows the coatings and deposition of thin films of metals with a control on the subnanometric scale. A quartz crystal sensor combined with a thin film controller (FTC-2000) guarantees an automatic control of the film thickness. Furthermore, a motorized rotation of the sample holder and the substrate and source shutter ensure a uniform deposition on the entire surface of the substrate. For the deposition of a homogeneous Au film, the sputtering rate was set to $6 \AA / \mathrm{s}$. We measured light transmission at $632.8 \mathrm{~nm}$ through the Au film and $50 \%$ of the incident light goes through. Finally, the AuNP substrate (iii) is prepared according to Refs. [31,32]. It is composed by oleylaminestabilized gold nanoparticles, $(12 \pm 2) \mathrm{nm}$ in diameter with an edge-to-edge interparticle separation of $\approx 10 \mathrm{~nm}$ between the particle surfaces, in a roughly hexagonal lattice, as shown in Fig. 1.

\section{B. Experimental setup}

The TERS system consists of a combination of a noncontact atomic force microscope (AFM) and a micro-Raman spectrometer. The micro-Raman system consists of an inverted microscope equipped with an oil immersion high numerical aperture objective (NA $=1.4$ ) responsible for focusing the excitation beam, as well as collecting the backscattered generated Raman signal, which is directed to a spectrometer [8]. Figure 2 illustrates the part of the system where the tip-sample coupling takes place, with the glass substrate in blue, the gold films in red, and the tip in yellow. The light travels from the bottom and, as such, the incident light crosses the glass coverslip, then the gold [when using

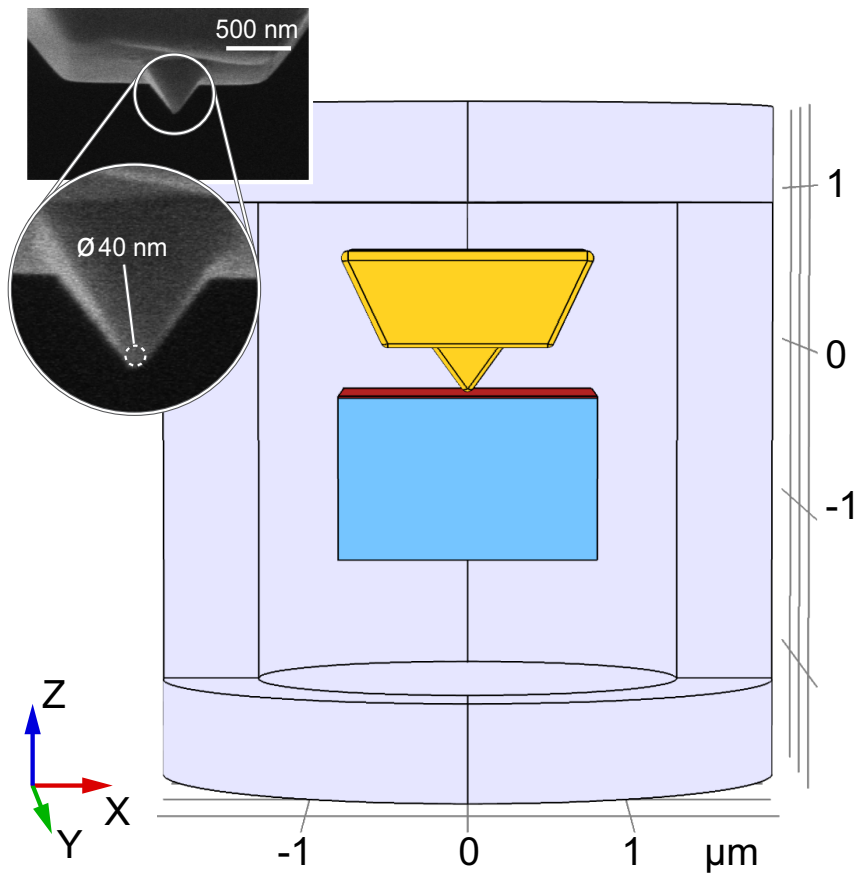

FIG. 2. Schematics of the tip-sample system. The PTTP is shown in yellow, the thin film on top of the substrate is shown in red and the glass substrate in light blue. The inset image portrays the SEM image of the actual tip used in some of the experiments with an indication of the apex's diameter. The simulation setup is enclosed by an air cylinder with $4.2 \mu \mathrm{m}$ height and $3.8 \mu \mathrm{m}$ diameter with 600 -nm-thick PMLs at the simulation boundaries.

substrates (ii) or (iii)], then the deposited graphene, reaching the TERS tip. The scattered light travels the way back. The inverted microscope oil immersion objective uses an oil that matches the index of refraction of the glass.

The AFM setup is a home-made shear-force system with a tuning fork operating at $32.8 \mathrm{kHz}$, associated with a phase-locked loop system that controls the tip-sample distance. To make a correct comparative analysis among the effect of different substrates on the TERS from graphene, similar tip-sample distances are guaranteed by a fixed AFM set point when the tip is engaged on the samples, i.e., $5 \mathrm{~nm}$ for all cases [33].

Considering that the TERS setup is based on a radially polarized He-Ne laser beam with a $632.8 \mathrm{~nm}$ wavelength, a resonant gold pyramidal tip, denominated plasmon-tunable tip pyramid (PTTP), was used (see inset to Fig. 2) [27]. The integrity of the radially polarized light after the sample, where the TERS nanoantenna is located, necessary for appropriated light-antenna coupling, is enabled by a Berek variable wave plate and checked with the assistance of a linear polarizer. The PTTP is capable of holding localized surface plasmon resonance, in this case tuned for the given excitation wavelength, so that the spectral enhancement is large enough to make the far-field contribution negligible when working with a tip-sample distance of $5 \mathrm{~nm}$ in the TERS configuration. In addition, the tip used has a $40 \pm 10 \mathrm{~nm}$ apex diameter, as measured by scanning electron microscopy (SEM, see inset to Fig. 2). 


\section{Field distribution simulations}

The simulations are based on the experimental setup described in Sec. II B. Figure 2 displays the positioning of tip and substrate in the simulation environment. The simulations were performed using the finite element method (FEM) implemented by the COMSOL MULTIPHYSICS $\mathrm{V}$ in the frequency domain. The tip utilized in the simulations was a PTTP tuned for a $632.8 \mathrm{~nm}$ excitation wavelength with an apex diameter of $40 \mathrm{~nm}$ and an internal angle of $70.54^{\circ}$ between pyramid faces. The boundaries are treated with a 600 -nm-thick perfectly matched layer (PML) in a cylindrical formulation as to adequately adapt to the simulated environment improving convergence. All the components not composed of air are not in contact with the PML to avoid calculation artifacts. Furthermore, the lateral size and thickness of the glass substrate are sufficient in order to avoid unexpected optical interference effects on the results. The tip-sample gap is set to $5 \mathrm{~nm}$ for all cases, as to properly simulate the gap for noncontact AFM and to take advantage of the light confinement [33]. The gold material model utilized for the PTTP tip, the gold film and the AuNP were obtained experimentally from reflection and transmission measurements of thin gold films by Johnson and Christy [34]. It is important to outline that, despite the nanometric metallic structures involved in the simulation, a nonlocal dielectric function $[35,36]$ was not considered in the simulations. For this reason, the absolute values for electric fields presented throughout the work are not considered in the analyses, which are all based on major effects on relative values obtained within the same incident and scattered excitation energies.

As for the input electromagnetic field, a radially polarized, tightly focused Gaussian beam was modeled using the paraxial approximation for a Gaussian beam with $360 \mathrm{~nm}$ waist diameter and polarization along the vertical axis (direction of propagation). Simulations start with an impute Gaussian beam waist whose diameter accounts for the central $Z$ lobe size in a system with a 1.4 numerical aperture (provided by an oil immersion objective lens) and a $632.8 \mathrm{~nm}$ excitation wavelength [33].

In order to reduce computational costs, the simulation environment was truncated at symmetry planes corresponding to $x=0 \mathrm{~nm}$ and $y=0 \mathrm{~nm}$, resulting in a quarter section of the original environment. The resulting new boundaries were treated as perfect magnetic conducting surfaces in order to impose symmetry to the electric field with respect to the cut planes.

Figures 3(a) and 3(b) describes the far-field (no tip) and Figs. 3(c)-3(f) near-field (with tip) intensity distributions obtained by the frequency-domain modeling, considering the outlined specifics of our experimental setup. The distinction between the field intensity distribution in the presence of glass or gold substrate is obtained, where the blue curves stand for nongap mode and the orange curves stand for the gap mode configurations in Figs. 3(a)-3(d). The left [(a) and (c)] and right [(b) and (d)] panels stand for the electric field polarization parallel $(X$, in-plane) and perpendicular $(Z$, outof-plane) to the substrate plane, respectively. In Figs. 3(e) and $3(\mathrm{f})$, the field vectors at the sample's plane are displayed as white arrows.
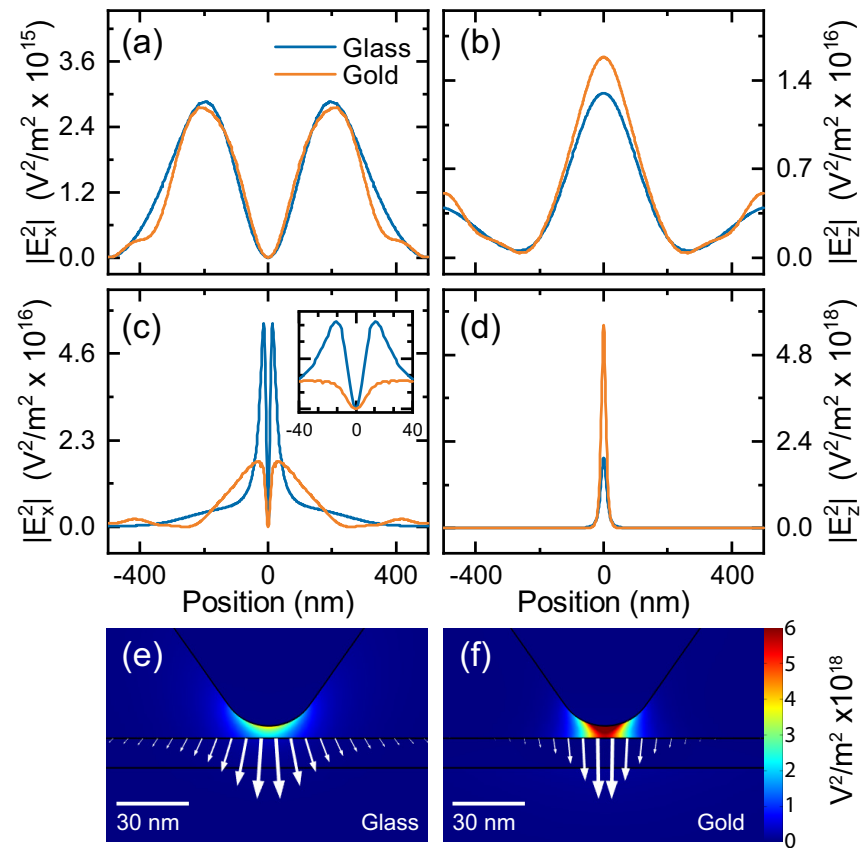

FIG. 3. Simulations of the electric field at the sample plane for glass and gold substrates. (a) and (b) show the intensity profile of the $\left|E_{x}^{2}\right|$ and $\left|E_{z}^{2}\right|$, respectively, in the absence of the tip; (c) and (d) display the field intensity at the same location, but with the tip at $5 \mathrm{~nm}$ distance from the substrate. The inset to (c) is a zoom up close to the tip location ( $0 \mathrm{~nm}$ position); (e) shows the color coded intensity map with electric field orientation at the glass substrate portrayed by white arrows and (f) is the equivalent result for the $12 \mathrm{~nm}$ gold film substrate.

Finally, we also performed two-dimensional simulations to understand super-resolution results obtained on the structured gap-mode configuration, where the computational costs get too high due to the loss of the square-lattice plasmonic symmetry. Further details on Sec. IV.

\section{Group theory analysis}

The TERS intensities depend not only on the incident field distribution, but also on the Raman tensors and scattering field intensities, which theoretically involve several possible processes, including spatial coherence [37]. We address these aspects in this paper from the symmetry standpoint, using group theory [38].

Considering graphene pertains to the $D_{6 h}$ point group, the $\mathrm{G}$ band, observed at $\approx 1584 \mathrm{~cm}^{-1}$ belongs to the $E_{2 g}$ irreducible representation, while the second-order $2 \mathrm{D}$ band (also known as G' band), observed at $\approx 2700 \mathrm{~cm}^{-1}$, is majorly a totally symmetric $A_{1 g}$ mode. The Raman tensors for the $\mathrm{G}$ and $2 \mathrm{D}$ bands of graphene, considering the presence of a highly focused field [39], are given by

$$
\alpha_{G}^{1}=\left(\begin{array}{ccc}
a & 0 & 0 \\
0 & -a & 0 \\
0 & 0 & 0
\end{array}\right), \quad \alpha_{G}^{2}=\left(\begin{array}{ccc}
0 & a & 0 \\
a & 0 & 0 \\
0 & 0 & 0
\end{array}\right),
$$


and

$$
\begin{aligned}
\alpha_{2 D}^{1} & =\left(\begin{array}{lll}
b & 0 & 0 \\
0 & b & 0 \\
0 & 0 & c
\end{array}\right), \quad \alpha_{2 D}^{2}=\frac{1}{4}\left(\begin{array}{ccc}
-b & -b \sqrt{3} & 0 \\
b \sqrt{3} & -b & 0 \\
0 & 0 & c
\end{array}\right), \\
\alpha_{2 D}^{3} & =\frac{1}{4}\left(\begin{array}{ccc}
-b & b \sqrt{3} & 0 \\
-b \sqrt{3} & -b & 0 \\
0 & 0 & c
\end{array}\right) .
\end{aligned}
$$

From symmetry, the $\mathrm{G}$ band can only be activated by electric fields in the graphene (XY) plane. The 2D band can also be activated by fields polarized perpendicular to the graphene plane $(\mathrm{Z})$. The $c$ value is not known in the literature, but experiments [39] indicate that $c \ll b$.

The selection rules for TERS have been derived by group theory [38]. The phonon active modes for the different scattering processes are defined by

$$
\begin{aligned}
\mathbf{S}:\left(\Gamma_{\mathrm{vec}} \otimes \Gamma_{\mathrm{vec}}\right) & \subset \Gamma_{p n}, \\
\mathbf{S P}:\left(\Gamma_{\mathrm{vec}} \otimes \Gamma^{\mathcal{H}_{\mathrm{plel}}} \otimes \Gamma_{\mathrm{vec}}\right) & \subset \Gamma_{p n}, \\
\mathbf{P S}:\left(\Gamma^{\mathcal{H}_{\mathrm{pl-el}}} \otimes \Gamma_{\mathrm{vec}}\right) & \subset \Gamma_{p n}, \\
\mathbf{P S P}:\left(\Gamma_{\mathrm{vec}} \otimes \Gamma^{\mathcal{H}_{\mathrm{pl}-\mathrm{el}}} \otimes \Gamma^{\mathcal{H}_{\mathrm{pl-el}}} \otimes \Gamma_{\mathrm{vec}}\right) & \subset \Gamma_{p n},
\end{aligned}
$$

where $\mathbf{S}$ is the usual Raman scattering Stokes process, where light interacts only with the sample; SP and PS are processes where the interaction of the incoming and outgoing light, respectively, is mediated by the plasmonic structure; PSP is a process where both incoming and outgoing light interactions are mediated by the plasmon. Notice Eq. (3c) is different from what has been presented in Ref. [38] because, in the case of a radially polarized incoming excitation (as utilized in the experimental setup described in Sec. II B), the PS lightinduced excitation of the plasmonic tip occurs via a totally symmetric field distribution rather than a vector-like linearly polarized excitation.

The difference in going from nongap mode to gap mode TERS is that the TERS system changes from the $C_{\infty v}$ point group to the $D_{\infty h}$ due to the mirror symmetry imposed by the metallic surface. When comparing regular TERS $\left(C_{\infty v}\right)$ with gap mode TERS $\left(D_{\infty h}\right)$, the PS scattering becomes forbidden for both the $\mathrm{G}$ and $2 \mathrm{D}$ bands in gap mode.

\section{EXPERIMENTAL RESULTS AND DISCUSSIONS}

\section{A. Tip scanning the diffraction limited confocal illumination area}

In conventional TERS setups, the AFM gold tip is aligned and fixed with respect to the laser focus, and the sample is moved along the XY plane by a piezo stage. In order to study the TERS spatial distribution when aligning the tip with the laser focus, we scanned the focal region in the XY plane by moving the tip with respect to the fixed laser spot (and sample), measuring the Raman signal intensity of graphene $2 \mathrm{D}$ band. This procedure was made for the graphene on glass (nongap mode) and for the graphene on top of the thin gold film (gap mode). By plotting the $2 \mathrm{D}$ band intensity as a function of tip position, we identify the spatial distribution of the convolution between near-field tip response and laser spot,
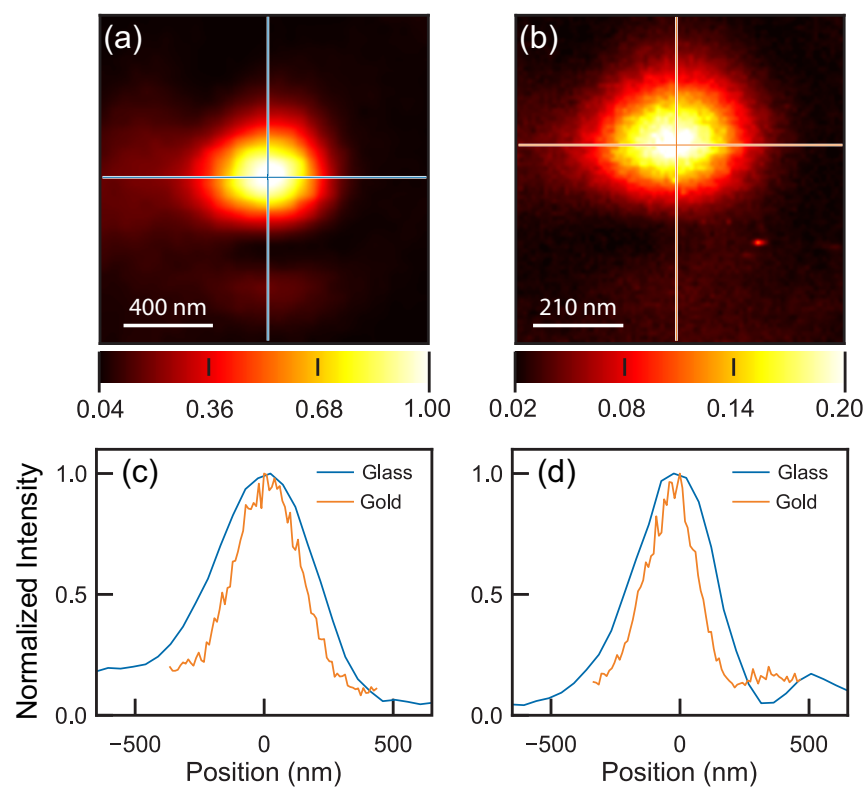

FIG. 4. Normalized 2D band TERS intensity for graphene as a function of tip position, when moving the tip across the laser illumination spot. The center position $(0 \mathrm{~nm})$ is the center of the laser spot, and the FWHM is an estimate of the diffraction limited (far-field) resolution. (a) graphene on glass; (b) graphene on gold film substrate (generated with the same tip), normalized by the same value as in (a); (c) and (d) show horizontal and vertical line profiles, respectively, for gold and glass substrates, from the locations indicated with corresponding colors in (a) and (b), but here with line profiles normalized to 1 .

as shown in Figs. 4(a) and 4(b). The maximum 2D band TERS intensity is obtained in the central $(0 \mathrm{~nm})$ position in both configurations. The full-width at half maximum (FWHM) is smaller in the gap mode configuration: $429 \mathrm{~nm}$ for the glass and $291 \mathrm{~nm}$ for gold, a 32\% reduction for the gap mode configuration. Similar (although less intense) results are observed for the $\mathrm{G}$ band TERS. These results provide a reasonable estimate of the size of the diffraction limited illumination area, and demonstrate a smooth distribution of the TERS signal in both glass and Au film substrate.

Figure 3 and 4 evidence different phenomena: the first only shows the incident electric field configuration, both without the tip [(a) and (b)] and with the tip positioned at the center of the illumination focal spot [(c)-(f)]; the second (Fig. 4) is the TERS intensity as a function of the probe position over the illumination focal spot. Still, the sharper TERS distribution for gap mode in Fig. 4 can be qualitatively understood based on the field distributions shown in Fig. 3. For the far field [Figs. 3(a) and 3(b)], there is a difference in the spread of the in-plane $X$-polarized field, which is slightly more compressed towards the center for the gap mode configuration, accompanied by a small increase in the very central intensity of the out-of-plane $Z$-polarized field. For the near-field configuration [Figs. 3(c) and 3(d)], the difference also depends on the direction of the electric field. For $Z$-polarized near-field (d), the field distribution in gap mode is $70 \%$ more intense and $26 \%$ narrower than for the nongap mode. For the $X$-polarized near field, Fig. 3(c), however, it is the opposite when looking 
TABLE I. $\mathrm{G}$ and 2D band spectral enhancement factors $F_{\mathrm{TERS}}=$ $A_{\mathrm{NF}} / A_{\mathrm{FF}}$ for the three substrate types.

\begin{tabular}{lccc}
\hline \hline & Glass & Au film & AuNP \\
\hline G & $10 \pm 4$ & $7 \pm 1$ & $5 \pm 3$ \\
2D & $16 \pm 1$ & $5 \pm 1$ & $5 \pm 1$ \\
\hline
\end{tabular}

closer to the central area under the tip, and the trend in the most intense signal exhibits inversions on each configuration (nongap mode versus gap mode, blue and orange curves, respectively) as the displacement from the central position increases. Overall, there is a sharper field distribution for the gap mode. The differences in TERS localization are even stronger considering that TERS intensity is proportional to electric field powers up to $|E|^{4}$ [16]. It is important to note, however, that, for this analysis, care has to be taken in proper alignment, since a change (maybe due to experimental drift) in the focus condition between these two experiments can also cause changes in the FWHM of the diffraction limited illumination spot.

\section{B. Near- and far-field comparison for different symmetry modes and different substrates}

We now analyze how different substrates influence the total spectral enhancement when the tip is placed in the optimal location for TERS signal, i.e., at position $0 \mathrm{~nm}$ in Figs. 4(c) and 4(d). The spectral enhancement factor is defined here as $F_{\mathrm{TERS}}=A_{\mathrm{NF}} / A_{\mathrm{FF}}$, where $A_{\mathrm{NF}}$ is the integrated intensity (area) of a Raman peak in the presence of the tip (NF standing for near field-with the tip down) and $A_{\mathrm{FF}}$ the equivalent value in the same region with the tip retracted far away from the sample (FF standing for far field-with the tip up). Figure 5 shows the graphene Raman spectra with and without the tip on the three different substrates.

The enhancement factors $F_{\text {TERS }}$ were measured for the $\mathrm{G}\left(E_{2 g}\right)$ and 2D $\left(A_{1 g}\right)$ bands on glass, Au film and AuNP substrates, resulting on the values summarized in Table I. An estimate of the local TERS enhancement factor can be obtained by multiplying $F_{\text {TERS }}$ by 100 , which is roughly the ratio between the focal laser spot area and TERS apex transversal area. However, be aware this is a rough estimate, since it does not consider the complexity of the field distribution (see also Ref. [40]).

The average results and the uncertainties were obtained analyzing seven tip up and seven tip down spectra like the ones shown in Fig. 5, obtained during a scanning procedure of homogeneous regions (accumulation time of 2 seconds per point for graphene on glass, 5 seconds for graphene on $\mathrm{Au}$ film, and 10 seconds for graphene on AuNP, excitation power of $160 \mu \mathrm{W}$ at the sample for all cases). The estimated uncertainty is larger for the $\mathrm{G}$ band on the AuNPs substrate because of the presence of the oleylamine peaks [see Fig. 5(c), near $1600 \mathrm{~cm}^{-1}$ ]. Interestingly, the enhancement factors change depending on the substrate and the Raman band. Counterintuitively, the overall enhancement is larger for regular TERS (on glass) as compared to the gap mode configurations, consistent with what has been shown in Figs. 4(a) and 4(b).

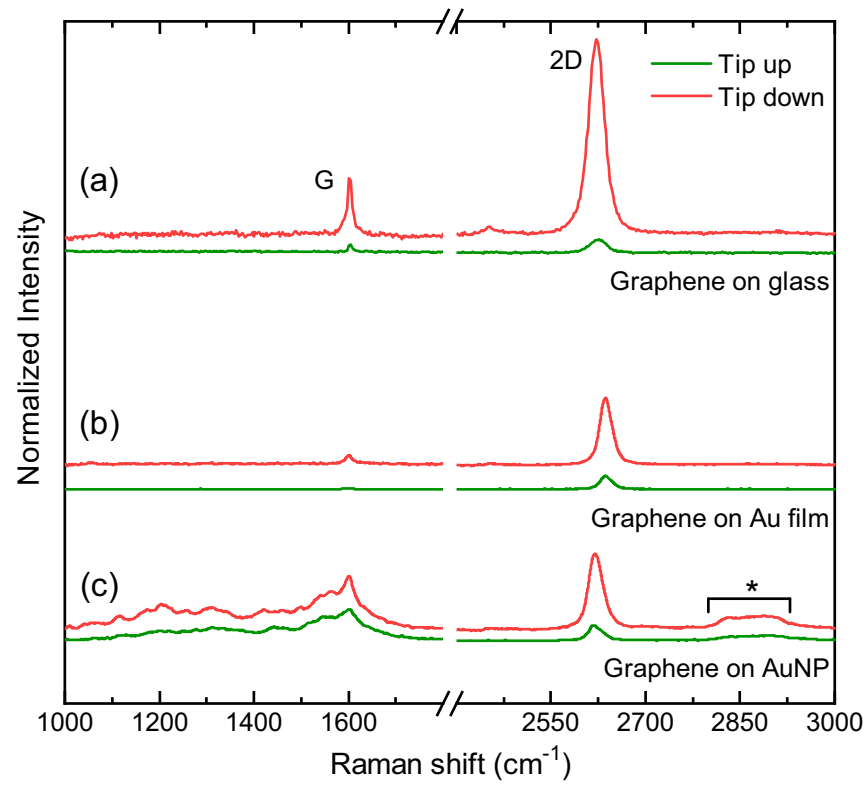

FIG. 5. Raman spectra of graphene placed on three different substrates [(a) glass; (b) Au film; (c) AuNP] in the spectral range of the $\mathrm{G}$ and 2D bands. Tip down and tip up spectra are marked as red and green, respectively. The tip down spectra are recorded when the tip is landed, i.e., $5 \mathrm{~nm}$ away from the sample, and the tip up spectra are recorded when the tip is lifted away (retracted more than $500 \mathrm{~nm}$ away from the sample by the SPM piezoelectric control mechanism, so that the tip has no effect and can be considered absent). All spectra are normalized to exhibit the same normalized 2D band amplitude on tip up condition and were acquired with the same excitation power. The $50 \%$ loss in intensity due to the $\mathrm{Au}$ film transmission is not accounted for in (b). The $*$ indicates the oleylamine feature utilized to plot Figs. 6(a) and 6(b).

This counterintuitive result can be understood based on the group theory analysis, combined with the electric field distributions shown in Fig. 3. The presence of the conductive substrate strongly enhances the $Z$-polarized field, but not the $X Y$-polarized fields. Since graphene responds to electric fields along the plane, the gap mode is actually not effective in enhancing the Raman response of this two-dimensional system. It is important to note that this result implies that the out-of-plane response of totally symmetric (2D) mode, although not symmetry forbidden, is truly negligible, i.e. the Raman tensor parameter $c \approx 0$ [see Eq. (2)]. As it can be seen in Table I, while on glass the enhancement factor of the 2D band intensity is 16 , on gold it is roughly three times smaller.

Besides, when comparing the results obtained for the $G$ $\left(E_{2 g}\right)$ and 2D $\left(A_{1 g}\right)$ modes, the result on glass is consistent with reports on the literature, where the 2D band enhances more than the $\mathrm{G}$ band due to near-field coherence effects that privilege totally symmetric modes [37,41]. Interestingly, this difference washes out in the gap mode configuration, and again, this can be understood as due to the stronger confinement of the field very near the tip location. The inset to Fig. 3(c) shows that, in the gap mode, the in-plane field is strongly reduced close to the tip location, within the phonon coherence length $(\sim 30 \mathrm{~nm})$, where the near-field interference 
(a)

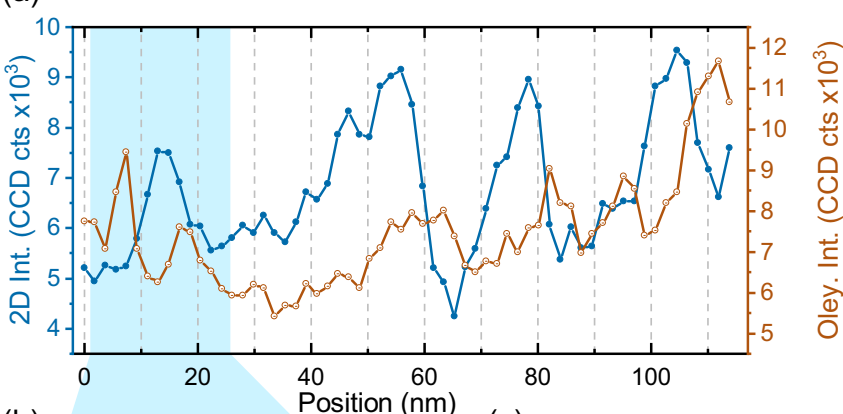

(b)

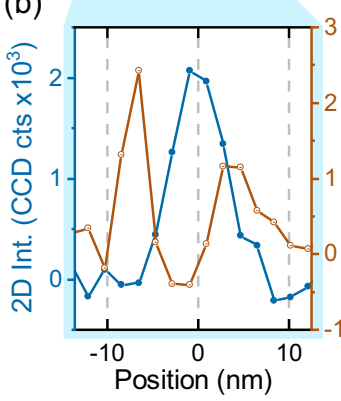

(c)

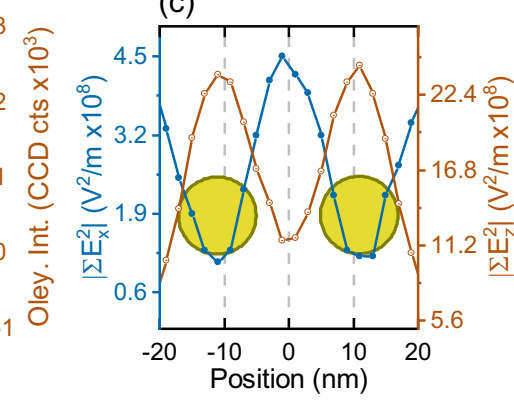

FIG. 6. (a) Intensity profile of the graphene 2D (blue filled bullets) and the oleylamine (orange open bullets) Raman peaks when moving the TERS tip along a line scan over AuNP (excitation power of $160 \mu \mathrm{W}$ and integration time of $2.5 \mathrm{~s}$ per point); (b) detail of section highlighted in (a) with linear backgrounds removed to improve visualization; (c) simulated intensities of integrated in-plane $(X$, blue) and out-of-plane ( $Z$, orange) electric field components along a line scan. The golden circles indicate the positions and sizes of the AuNP in the simulation.

effects take place [41]. This also confirms that the higher enhancement for the 2D band on glass is due to the nonlocal PS and SP scattering [37,38], rather than due to the outof-plane $c$ component of the Raman tensor, otherwise the 2D band should enhance more (not less) in gap mode (see Table I).

\section{TERS line profile in structured gap mode}

To test field localization and the possible achievement of ultrahigh resolution, we measured graphene on top of the AuNP substrate, as described in Sec. II A, while scanning the substrate. Since graphene is homogeneously present in this sample, the only variation throughout the scan is the configuration of AuNP underneath the tip's apex. Figure 6(a) shows the intensity trends of the 2D phonon mode (blue filled bullets) and also the intensity of a nearby Raman band [orange open bullets, $\approx 2850 \mathrm{~cm}^{-1}$, see $*$ in Fig. 5(c)], attributed to oleylamine, during the line scan. Since the AuNPs are coated by a layer of oleylamine, required for the self-assembly into an AuNP monolayer, its Raman band can also be observed.

Considering that the tip used for the experiment shown in Fig. 6 has a $40 \mathrm{~nm}$ apex diameter, the total scan of $120 \mathrm{~nm}$ is a relatively small scanning region. Still, clear oscillations in the 2D and oleylamine Raman intensities are observed. In terms of lateral resolution, a fast Fourier transform analysis of the 2D band intensity map from which the line profile in Fig. 6(a)

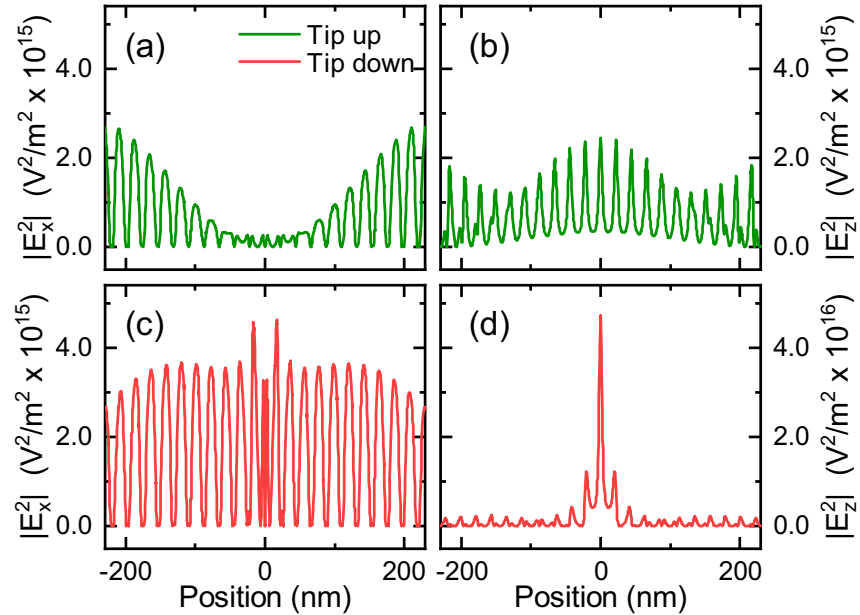

FIG. 7. Simulated tip up [(a) and (b)] and down [(c) and (d)] distributions for in-plane [(a) and (c)] and out-of-plane [(b) and (d)] squared electric field components. To probe the field distribution, the values of the electric field were plotted over a horizontal line $4.9 \mathrm{~nm}$ away from the tip's apex and $0.1 \mathrm{~nm}$ away the nanospheres, which is roughly were the graphene would be located.

was taken, results in a spatial resolution of $6.7 \mathrm{~nm}$, close to the Nyquist limit of $3.75 \mathrm{~nm}$ expected for the $1.875 \mathrm{~nm}$ per pixel sampling rate utilized. This can be considered superresolution given the $40 \pm 10 \mathrm{~nm}$ apex diameter for the tip utilized in this experiment.

Interestingly, we observe that whenever the intensity of the $2 \mathrm{D}$ band increases, the intensity of the oleylamine band decreases. The alternating peak intensity locations when comparing the 2D band and oleylamine bands can be explained considering the intensity profile trends of the in-plane $(X)$ and out-of-plane $(Z)$ components of the electric field as the sample is scanned, as shown in the simulation results in Fig. 6(c) (more detail in Sec. IV). Note the similarity between the simulation [Fig. 6(c)] and the detailed experimental section in Fig. 6(b). The 2D band is maximum when the in-plane $X$ field is maximum, which happens when the tip apex is between particles, while the oleylamine peaks are maximum when the out-of-plane $Z$ field is maximum, which happens when the tip apex is on top of a particle.

\section{FURTHER SIMULATIONS AND DISCUSSIONS ON SUPER-RESOLUTION}

Section III C showcases how increased resolution can be obtained from a structured gap mode substrate. In this section, frequency domain simulations using an adapted version of the setup described in Sec. II C, limited to two dimensions are used in order to properly characterize the field distribution when "super-resolution" situation is achieved. In the simulation environment, the substrate is modeled by 50 gold circles with a diameter of $12 \mathrm{~nm}$ and a $10 \mathrm{~nm}$ gap between each other.

Figure 7 shows a simulated tip up and down experiment. For this structured substrate, the profiles observed in Figs. 3(a)-3(d) are now superposed by modulations induced by the AuNP. When the tip is landed (red traces), there is 


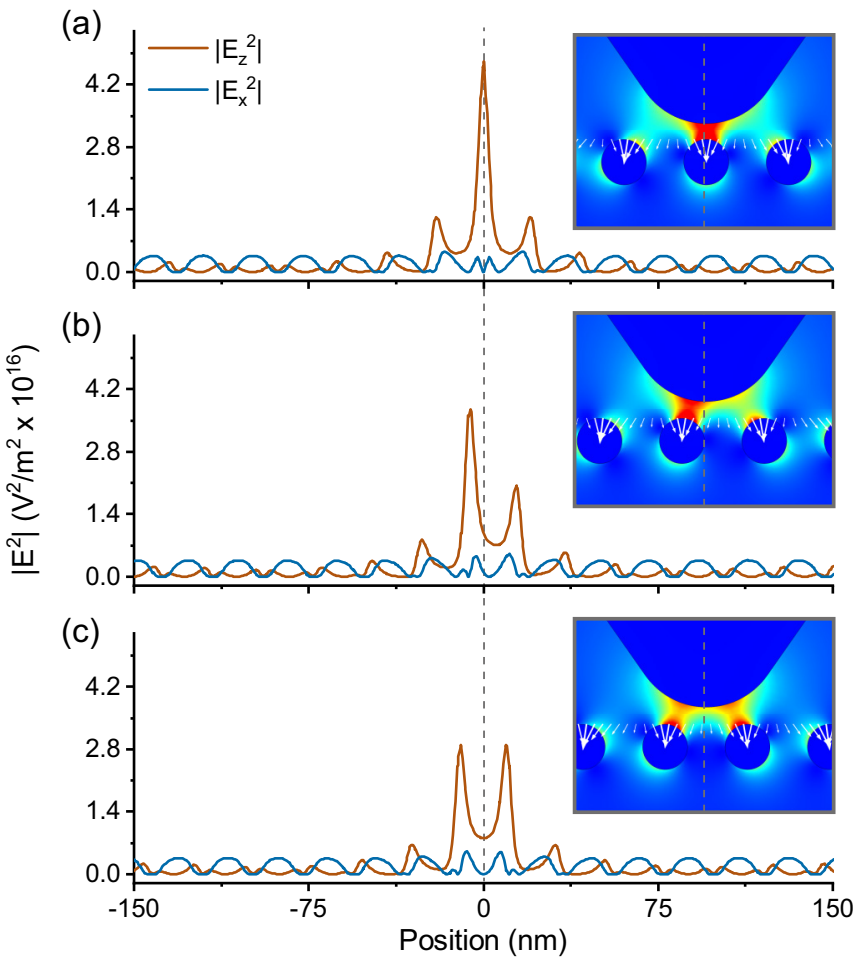

FIG. 8. Electric field intensity distribution for in-plane and outof-plane components for distinct particle configurations under the tip: (a) tip on top of a particle, (b) slightly misaligned with a particle, and (c) in between particles. The fields are plotted over the same region described in Fig. 7. The insets to each graph portray a color coded two-dimensional distribution of $|E|^{2}$.

an increase in field intensity. However, the enhancement is localized near the tip for the out-of-plane $Z$-field component [Fig. 7(d)], but completely delocalized for the in-plane $X$-field component [Fig. 7(c)]. Therefore, for the experiment shown in Figs. 6(a) and 6(b), while the oleylamine spectra comes majorly from molecules localized under the tip, the picture is completely different for the graphene 2D band.

For further details, Fig. 8 shows the theoretical changes in the $X$ - and $Z$-field intensities for different relative position of the tip with respect to the AuNPs, i.e., right on top of a particle (a), exactly in between two particles (c), and between these two cases (b). In all cases, the graphene TERS signal (given by the $X$-polarized field) should come from the entire focal region, while some degree of localization is only obtained for TERS related to the $Z$-polarized field component.

\section{CONCLUSIONS}

By exploring experimentally and theoretically the TERS electric field distribution in graphene on different substrates, we consistently found that in gap mode configuration a strong $Z$-polarized field is achieved, but it does not generate extra enhancement for two-dimensional systems such as graphene, which responds to electric fields polarized along the substrate plane. Our analysis solidifies the conclusion that the totally symmetric modes in graphene have a negligible Raman response for fields polarized perpendicular to the graphene plane, even if not symmetry forbidden. Furthermore, we show that near-field interference effects are significantly suppressed for the in-plane fields in gap mode.

Additionally, it was shown, both by simulations and experiments, that the composition of the substrate (glass or gold) and its structure (gold film or gold nanoparticles) have an effect on field distribution and, consequently, on resolution. Nevertheless, the resolution can be further improved, beyond the tip's apex diameter, by means of a careful choice of the tip-sample-substrate interaction. For instance, a conductive substrate with features smaller than the tip's apex, such as gold nanoparticles, can be employed to improve the lateral resolution, although this is effective only for the out-of-plane polarized field. The substrate actually delocalizes the in-plane electric field. This effect must be carefully considered when analyzing subnanometer TERS measurements, as the tip will still interact with all subnanometer features in its vicinity. Although our results were developed for nanometer-sized structures, similar effects should be observed in picocavity measurements [22,23].

\section{ACKNOWLEDGMENTS}

The authors acknowledge technical support from Rafael Nadas. This work was supported by the Fundação Coordenação de Aperfeiçoamento de Pessoal de Nível (CAPES) and the Deutsche Akademische Austauschdienst (DAAD) within the PROBRAL program under Grant number 57446501. A.J. acknowledges financial support from the Humboldt Foundation and CNPq (552124/2011-7,307481/2013-1, 304869/2014-7, 460045/2014-8, 305384/2015-5, 309861/2015-2. H.M. and C.R. acknowledges financial support from Finep, $\mathrm{CNPq}$ and Fapemig. H.L. acknowledges the German Research Foundation (DFG) for financial support via the Cluster of Excellence "Advanced Imaging of Matter" (EXC 2056 - Project No. 390715994). P.K. and S.R. acknowledge support by the European Research Council ERC under Grant DarkSERS (772108) and the Focus Area NanoScale of Freie Universität.
[1] J. Wessel, JOSA B 2, 1538 (1985).

[2] R. M. Stöckle, Y. D. Suh, V. Deckert, and R. Zenobi, Chem. Phys. Lett. 318, 131 (2000).

[3] A. Hartschuh, E. J. Sánchez, X. S. Xie, and L. Novotny, Phys. Rev. Lett. 90, 095503 (2003).

[4] A. Hartschuh, N. Anderson, and L. Novotny, J. Microscopy 210, 234 (2003).
[5] A. Hagen, M. Steiner, M. B. Raschke, C. Lienau, T. Hertel, H. Qian, A. J. Meixner, and A. Hartschuh, Phys. Rev. Lett. 95, 197401 (2005).

[6] I. O. Maciel, N. Anderson, M. A. Pimenta, A. Hartschuh, H. Qian, M. Terrones, H. Terrones, J. Campos-Delgado, A. M. Rao, L. Novotny, and A. Jorio, Nat. Mater. 7, 878 (2008).

[7] E. Bailo and V. Deckert, Chem. Soc. Rev. 37, 921 (2008). 
[8] L. G. Cançado, A. Hartschuh, and L. Novotny, J. Raman Spectrosc. 40, 1420 (2009).

[9] B.-S. Yeo, J. Stadler, T. Schmid, R. Zenobi, and W. Zhang, Chem. Phys. Lett. 472, 1 (2009).

[10] T. A. Yano, P. Verma, Y. Saito, T. Ichimura, and S. Kawata, Nat. Photonics 3, 473 (2009).

[11] E. M. Van Schrojenstein Lantman, T. Deckert-Gaudig, A. J. Mank, V. Deckert, and B. M. Weckhuysen, Nat. Nanotechnol. 7, 583 (2012).

[12] N. Kumar, B. Stephanidis, R. Zenobi, A. J. Wain, and D. Roy, Nanoscale 7, 7133 (2015).

[13] K.-D. Park, O. Khatib, V. Kravtsov, G. Clark, X. Xu, and M. B. Raschke, Nano Lett. 16, 2621 (2016).

[14] X. Wang, S.-C. Huang, T.-X. Huang, H.-S. Su, J.-H. Zhong, Z.-C. Zeng, M.-H. Li, and B. Ren, Chem. Soc. Rev. 46, 4020 (2017).

[15] R. Beams, J. Raman Spectrosc. 49, 157 (2018).

[16] Z. Yang, J. Aizpurua, and H. Xu, J. Raman Spectrosc. 40, 1343 (2009).

[17] V. Deckert, J. Raman Spectrosc. 40, 1336 (2009).

[18] X. Shi, N. Coca-López, J. Janik, and A. Hartschuh, Chem. Rev. 117, 4945 (2017).

[19] J. Langer, D. Jimenez de Aberasturi, J. Aizpurua, R. A. AlvarezPuebla, B. Auguié, J. J. Baumberg, G. C. Bazan, S. E. J. Bell, A. Boisen, A. G. Brolo, J. Choo, D. Cialla-May, V. Deckert, L. Fabris, K. Faulds, F. J. García de Abajo, R. Goodacre, D. Graham, A. J. Haes, C. L. Haynes, C. Huck, T. Itoh, M. Käll, J. Kneipp, N. A. Kotov, H. Kuang, E. C. Le Ru, H. K. Lee, J.-F. Li, X. Y. Ling, S. A. Maier, T. Mayerhöfer, M. Moskovits, K. Murakoshi, J.-M. Nam, S. Nie, Y. Ozaki, I. Pastoriza-Santos, J. Perez-Juste, J. Popp, A. Pucci, S. Reich, B. Ren, G. C. Schatz, T. Shegai, S. Schlücker, L.-L. Tay, K. G. Thomas, Z.-Q. Tian, R. P. Van Duyne, T. Vo-Dinh, Y. Wang, K. A. Willets, C. Xu, H. Xu, Y. Xu, Y. S. Yamamoto, B. Zhao, and L. M. Liz-Marzán, ACS Nano 14, 28 (2020).

[20] C. Chen, N. Hayazawa, and S. Kawata, Nat. Commun. 5, 3312 (2014).

[21] R. Zhang, Y. Zhang, Z. Dong, S. Jiang, C. Zhang, L. Chen, L. Zhang, Y. Liao, J. Aizpurua, and Y. e. Luo, Nature (London) 498, 82 (2013).

[22] J. Lee, K. T. Crampton, N. Tallarida, and V. A. Apkarian, Nature (London) 568, 78 (2019).

[23] J. J. Baumberg, J. Aizpurua, M. H. Mikkelsen, and D. R. Smith, Nat. Mater. 18, 668 (2019).

[24] W. Zhang, B. S. Yeo, T. Schmid, and R. Zenobi, J. Phys. Chem. C 111, 1733 (2007).
[25] J. Stadler, B. Oswald, T. Schmid, and R. Zenobi, J. Raman Spectrosc. 44, 227 (2013).

[26] T. L. Vasconcelos, B. S. Archanjo, B. Fragneaud, B. S. Oliveira, J. Riikonen, C. Li, D. S. Ribeiro, C. Rabelo, W. N. Rodrigues, A. Jorio, C. A. Achete, and L. G. Cançado, ACS Nano 9, 6297 (2015).

[27] T. L. Vasconcelos, B. S. Archanjo, B. S. Oliveira, R. Valaski, R. C. Cordeiro, H. G. Medeiros, C. Rabelo, A. Ribeiro, P. Ercius, C. A. Achete, A. Jorio, and L. G. Cançado, Adv. Opt. Mater. 6, 1800528 (2018).

[28] S. F. Becker, M. Esmann, K. W. Yoo, P. Gross, R. Vogelgesang, N. K. Park, and C. Lienau, ACS Photonics 3, 223 (2016).

[29] A. Jorio, R. Saito, G. Dresselhaus, and M. S. Dresselhaus, Raman Spectroscopy in Graphene Related Systems (Wiley-VCH Verlag GmbH \& Co. KGaA, Weinheim, Germany, 2011).

[30] A. Jorio, L. G. Cançado, S. Heeg, L. Novotny, and A. Hartschuh, in Handbook of Carbon Nanomaterials, 1st ed., edited by R. B. Weisman and J. Kono (World Scientific, 2019), Chap. 5, pp. 175-221.

[31] F. Schulz, S. Tober, and H. Lange, Langmuir 33, 14437 (2017).

[32] N. S. Mueller, B. G. Vieira, F. Schulz, P. Kusch, V. Oddone, E. B. Barros, H. Lange, and S. Reich, ACS Photonics 5, 3962 (2018).

[33] L. Novotny and B. Hecht, Principles of Nano-Optics (Cambridge University Press, Cambridge, 2012).

[34] P. B. Johnson and R. W. Christy, Phys. Rev. B 6, 4370 (1972); L. Vinet and A. Zhedanov, J. Phys. A: Math. Theor. 44, 085201 (2011).

[35] J. M. McMahon, S. K. Gray, and G. C. Schatz, Phys. Rev. Lett. 103, 097403 (2009).

[36] J. M. McMahon, S. K. Gray, and G. C. Schatz, Phys. Rev. B 82 035423 (2010).

[37] L. G. Cançado, R. Beams, A. Jorio, and L. Novotny, Phys. Rev. X 4, 031054 (2014).

[38] A. Jorio, N. S. Mueller, and S. Reich, Phys. Rev. B 95, 155409 (2017).

[39] H. Budde, N. Coca-López, X. Shi, R. Ciesielski, A. Lombardo, D. Yoon, A. C. Ferrari, and A. Hartschuh, ACS Nano 10, 1756 (2016).

[40] A. R. Neto, C. Rabelo, L. G. Cancado, M. Engel, M. Steiner, and A. Jorio, in 2019 4th International Symposium on Instrumentation Systems, Circuits and Transducers (INSCIT), Vol. 3 (IEEE, 2019), pp. 1-6.

[41] R. Beams, L. G. Cançado, S.-H. Oh, A. Jorio, and L. Novotny, Phys. Rev. Lett. 113, 186101 (2014). 